\title{
Texture Analysis Based on Pi-Rads 4/5-Scored MRI Images Combined with Machine Learning to Distinguish Benign Lesions from Prostate Cancer
}

\section{Lu Ma}

First Affiliated Hospital of Soochow University

\section{Qi Zhou}

The First Affiliated Hospital of Soochow University

Huming Yin

The First Affiliated Hospital of Soochow University

\section{Xiaojie Ang}

The First Affiliated Hospital of Soochow University

Yu Li

The First Affiliated Hospital of Soochow University

\section{Gansheng Xie}

The First Affiliated Hospital of Soochow University

\section{Gang Li ( $\nabla$ ganglisdfyy@163.com )}

The First Affiliated Hospital of Soochow University

\section{Research Article}

Keywords: Prostate cancer, Radiomics, Texture feature analysis, Machine learning, PI-RADS score

Posted Date: August 26th, 2021

DOl: https://doi.org/10.21203/rs.3.rs-785959/v1

License: (9) This work is licensed under a Creative Commons Attribution 4.0 International License. Read Full License 


\section{Abstract}

Background: To extract the texture features of Apparent Diffusion Coefficient (ADC) images in Mp-MRI and build a machine learning model based on radiomics texture analysis to determine its ability to distinguish benign from prostate cancer (PCa) lesions using PI-RADS 4/5 score.

Materials and methods: First, use ImageJ software to obtain texture feature parameters based on ADC images; use $\mathrm{R}$ language to standardize texture feature parameters, and use Lasso regression to reduce the dimensionality of multiple feature parameters; then, use the feature parameters after dimensionality reduction to construct image-based groups. Learn R-Logistic, R-SVM, R-AdaBoost to identify the machine learning classification model of prostate benign and malignant nodules. Secondly, the clinical indicators of the patients were statistically analyzed, and the three clinical indicators with the largest AUC values were selected to establish a classification model based on clinical indicators of benign and malignant prostate nodules. Finally, compare the performance of the model based on radiomics texture features and clinical indicators to identify benign and malignant prostate nodules in PI-RADS 4/5.

Results: The experimental results show that the AUC of the R-Logistic model test set is 0.838 , which is higher than the R-SVM and R-AdaBoost classification models. At this time, the corresponding R-Logistic classification model formula is: Y_radiomics $=9.396-7.464 *$ median ADC-0.584

*kurtosis+0.627*skewness+0.576*MRI lesions volume; analysis of clinical indicators shows that the 3 indicators with the highest discrimination efficiency are PSA, Fib, LDL-C, and the corresponding C-Logistic classification model formula is: Y_clinical $=-2.608+0.324 * P S A-3.045 * F i b+4.147 * L D L-C$, the AUC value of the model training set is 0.860 , which is smaller than the training set R-Logistic classification model AUC value of 0.936 .

Conclusion: The machine learning classifier model is established based on the texture features of radiomics. It has a good classification performance in identifying benign and malignant nodules of the prostate in PI-RADS 4/5. This has certain potential and clinical value for patients with prostate cancer to adopt different treatment methods and prognosis.

\section{Background}

Prostate cancer ( $\mathrm{PCa}$ ) is a common malignancy among adult men in the United States. According to the national malignant tumor survey results, the incidence of $\mathrm{PCa}$ is $63.4 \%$, and the fatality rate has reached $26.6 \%{ }^{[1]}$. Studies found that PCa ranks third behind lung cancer and colorectal cancer for causes of death among American men ${ }^{[2,3]}$. Recently, the mortality rate among new cases with PCa in China has increased sharply. The primary pathogenic factors were family member genetics, age, and living habits. Currently, it is the fastest-growing malignancy in men, primarily occurring in middle-aged and older men ${ }^{[4]}$.

The diagnosis methods for PCa include digital rectal examination (DRE), TRUS, prostate specific antigen (PSA), Magnetic Resonance Imaging (MRI), and prostate biopsy. TRUS can obtain images from selected biopsy sites but cannot accurately detect all areas affected by PCa. Although the pathologic specificity of 
PSA screening for $\mathrm{PCa}$ is relatively high, factors such as benign prostatic hyperplasia, urinary retention, prostate massage, and frequent sexual activity also increase PSA levels, and patients with relatively high PSA levels still require a prostate biopsy to establish the diagnosis ${ }^{[5]}$. The early diagnosis of PCa determines the treatment and outcome; therefore, it is essential to identify simple, effective, accurate, and specific methods.

With the development of medical imaging technology, the role of MRI in clinical practice has extended to various stages, including tumor detection, disease monitoring during active surveillance, and sequential imaging for follow-up. It is one of the important methods for clinical diagnosis of PCa at present ${ }^{[6,7]}$. The Prostate Imaging Reporting and Data System (PI-RADS v2) is a standardized prostate Mp-MRI solution that combines anatomical T2-weighted images with one or more functional sequences, DWI, and dynamic contrast enhancement (DCE) sequences.

Radiomics can capture the properties of tissues and lesions, including the shape and heterogeneity of the tumor. Much mining texture feature data can be extracted from conventional medical examination images with high throughput through a computer-aided diagnosis system. Studies showed that radiomics texture features are associated with the heterogeneity index at the cellular level ${ }^{[8]}$. The application of this method in PCa enables the automatic localization of the disease and provides a noninvasive solution to evaluate the biological characteristics of the tumor. Some investigators have used it to predict $\mathrm{PCa}{ }^{[9]}$.

Machine learning $(\mathrm{ML})$ regressions have been widely used ${ }^{[10-12]}$. Their main advantage lies in their ability to analyze large amounts of data ${ }^{[13]}$. ML methods automatically collect selective clinical disease information from large-scale data, and this feature has proven to carry great value for diagnosis, classification, outcome, and other aspects. The construction of a disease prediction or classification model based on ML regressions streamlines auxiliary diagnosis and early risk warning, helps clinicians make efficient diagnoses, reduces time and costs, and reduces the risks associated with a needle biopsy.

Relevant studies focused primarily on clinical diagnosis and prognosis prediction of prostate cancer through Mp-MRI combined with radiomics and ML in artificial intelligence ${ }^{[14-18]}$. However, at present, there has been less study of prostate imaging texture analysis, PI-RADS V2 score, and clinical indicators combined with machine learning regressions to build a prediction model for differentiating benign and malignant lesions of PCa.

Therefore, this study was based on the PI-RADS V2 version ${ }^{[19]}$. Patients with PI-RADS V2 score $4 / 5$ were selected, and three ML methods (SVM, logistic regression, and AdaBoost) were applied. The models were analyzed on receiver operating characteristic curves (ROCs), and the performance of each prediction model was judged by comparing the AUCs. We did this to provide a reference model for the discrimination of benign from malignant prostatic lesions in patients with PI-RADS 4/5 score and to provide a new research approach to reduce overtreatment. 


\section{Materials And Methods \\ 2.1 Selection of clinical cases}

The subjects were clinical imaging data of 250 patients who underwent TRUS/MRI-targeted prostate biopsy at the First Affiliated Hospital of Soochow University from August 2017 to January 2020. Indicators included body mass index $\left(\mathrm{kg} / \mathrm{m}^{2}\right), P S A(\mathrm{ng} / \mathrm{mL})$, free prostate-specific antigen $(\mathrm{ng} / \mathrm{mL})$, white blood cells $\left(10^{9} / \mathrm{L}\right)$, fibrinogen $(\mathrm{g} / \mathrm{L})$, albumin $(\mathrm{g} / \mathrm{L})$, neutrophil-lymphocyte ratio, C-reactive protein $(\mathrm{mg} / \mathrm{L})$, platelet count $\left(10^{9} / \mathrm{L}\right)$, low-density lipid protein cholesterol $(\mathrm{mg} / \mathrm{dL})$, high-density lipoprotein cholesterol $(\mathrm{mg} / \mathrm{dL})$, and triglyceride ( $\mathrm{mol} / \mathrm{L})$. According to the inclusion criteria in Table 1, 103 case samples were collected, including 20 cases in the BPH group and 83 cases in the PCa group, with a median age of 71 years $(70.32 \pm 8.81$ years). We deleted the case data in which the pathological description area did not match the results of MR images or where the whole tumor boundary could not be accurately delineated.

Table 1

Standards of Case Selection

\section{Case Tips \\ Selection}

Inclusion MP-MRI scan was performed before surgery;

criteria

The PI-RADS V2 score was 4/5;

Prostatic biopsy results confirmed prostatic hyperplasia or prostate cancer

Exclusion Patients with contraindication of MRI examination;

criteria

The quality of MRI images was not good, or the images are incomplete and cannot be analyzed;

Patients with prostatitis, acute infectious diseases, hematological diseases, and other diseases that may cause changes in systemic inflammatory indicators

\subsection{Methods}

\subsubsection{Pathological grouping}

All patients underwent TRUS/MRI-targeted biopsy to obtain pathological results. Tissue specimens were fixed with $10 \%$ formalin, sections were embedded in paraffin, and routine HE staining was performed. The films were read by senior pathologists and confirmed by the pathological diagnosis as BPH or PCa.

Twenty cases were in the BPH group, and 83 cases were in the PCa group ${ }^{[20]}$.

\subsubsection{Acquisition and processing of MRI images}


All patients were examined using 3.0T Mp-MRI (Siemens Skyra) and 8-channel phased-front abdominal signal reception, including the prostate gland and bilateral seminal vesicles. The parameters are shown in Table 2.

Table 2

The parameters of MP-MRI scan sequence

\begin{tabular}{|lllllll|}
\hline Mp-MRI & Sequence & TR/TE(ms) & $\begin{array}{l}\text { distance factor } \\
(\mathrm{ms})\end{array}$ & NSA & $\begin{array}{l}\text { FON } \\
(\mathbf{m m})\end{array}$ & Matrix \\
\hline T1WI & TSE & $700 / 13$ & $5 / 0.5$ & 1 & $320 \times 250$ & $0.5 \times 0.5 \times 3$ \\
\hline T2WI & TSE & $4000 / 89$ & $3 / 0$ & 3 & $640 \times 640$ & $0.5 \times 0.5 \times 3$ \\
\hline $\begin{array}{l}\text { DWI (b =800, 1000, } \\
\text { 2000) }\end{array}$ & 2D-EPI & $4000 / 56$ & $3 / 0$ & 8 & $160 \times 296$ & $1.3 \times 1.3 \times 3$ \\
\hline T1WI-DCE & TWIST & $3.22 / 1.18$ & $3 / 0$ & 1 & $512 \times 512$ & $1.4 \times 1.4 \times 3$ \\
\hline
\end{tabular}

\begin{tabular}{|c|c|c|c|c|c|c|}
\hline Mp-MRI & Sequence & $\begin{array}{l}\text { TR/TE } \\
\text { (ms) }\end{array}$ & $\begin{array}{l}\text { distance factor } \\
\text { (ms) }\end{array}$ & NSA & $\begin{array}{l}\text { FON } \\
(\mathrm{mm})\end{array}$ & Matrix \\
\hline T1WI & TSE & $700 / 13$ & $5 / 0.5$ & 1 & $320 \times 250$ & $0.5 \times 0.5 \times 3$ \\
\hline T2WI & TSE & $4000 / 89$ & $3 / 0$ & 3 & $640 \times 640$ & $0.5 \times 0.5 \times 3$ \\
\hline $\begin{array}{l}\text { DWI }(b=800,1000, \\
2000)\end{array}$ & 2D-EPI & $4000 / 56$ & $3 / 0$ & 8 & $160 \times 296$ & $1.3 \times 1.3 \times 3$ \\
\hline T1WI-DCE & TWIST & $3.22 / 1.18$ & $3 / 0$ & 1 & $512 \times 512$ & $1.4 \times 1.4 \times 3$ \\
\hline \multicolumn{7}{|c|}{$\begin{array}{l}\text { All MRI images were downloaded from the PACS system in DICOM format from conventional T2WI, } \\
\text { DWI, and DCE-MRI for subsequent processing. The results of the PI-RADS V2 score were evaluated by } \\
\text { two professionally trained radiologists and were scored strictly according to the PI-RADS V2 score } \\
\text { criteria: } 1 \text { - very low likelihood of cancer; } 2 \text { - low likelihood of cancer; } 3-\text { moderate likelihood of } \\
\text { cancer; } 4 \text { - high likelihood of cancer; and } 5 \text { - very high likelihood of cancer. The scoring criteria of PI- } \\
\text { RADS v2 are shown in Table } 3-7 \text {. The scoring criteria of PI-RADS v2 are shown in Table } 3-7 \text {. }\end{array}$} \\
\hline
\end{tabular}


Table 3

PI-RADS V2 T2WI score

\begin{tabular}{|c|c|}
\hline $\begin{array}{l}\text { T2WI } \\
\text { (Peripher } \\
\text { Band) }\end{array}$ & standards of grading \\
\hline 1 score & The signal intensity is uniform and high \\
\hline 2 score & Linear, wedge-shaped or diffuse mild low signal with unclear boundary \\
\hline 3 score & $\begin{array}{l}\text { The signal intensity is not uniform or the boundary is not clear, is round, medium low } \\
\text { signal, including other does not meet } 2,4 \text { or } 5 \text { points standard }\end{array}$ \\
\hline 4 score & $\begin{array}{l}\text { Confined to the prostate, well defined, uniformly moderately low, signal lesion or mass, } \\
\text { maximum diameter }<1.5 \mathrm{~cm}\end{array}$ \\
\hline 5 score & $\begin{array}{l}\text { The imaging findings were the same as those of } 4 \text { points, but the maximum diameter } \\
\text { was more than or equal to } 1.5 \mathrm{~cm} \text {, or there was a clear extension or invasion to the } \\
\text { prostate }\end{array}$ \\
\hline \multicolumn{2}{|l|}{ T2WI } \\
\hline \multicolumn{2}{|c|}{$\begin{array}{l}\text { (transition } \\
\text { zone) }\end{array}$} \\
\hline 1 score & Uniform medium signal strength (normal) \\
\hline 2 score & Localized hypointense or inhomogeneous enveloped lesions (prostatic hyperplasia) \\
\hline 3 score & $\begin{array}{l}\text { Blurred edges, uneven signal strength, including others that do not meet } 2,4 \text {, or } 5 \text { score } \\
\text { criteria }\end{array}$ \\
\hline 4 score & Lenticular or ill-defined, uniform moderately low signal, maximum diameter $<1.5 \mathrm{~cm}$ \\
\hline 5 score & $\begin{array}{l}\text { Imaging findings were the same as } 4 \text { points, but the maximum diameter was } \geq 1.5 \mathrm{~cm} \text {, } \\
\text { or there was definite extension or invasion out of the prostate }\end{array}$ \\
\hline
\end{tabular}


Table 4

PI-RADS v2 DWI score

\section{DWI standards of grading}

$1 \quad$ No abnormalities were found on ADC and high B-value images.

score

$2 \quad$ ADC diagram fuzzy low signal.

score

3 Focal light to moderate low signal intensity on $A D C$ images, and equal to mild high signal

score intensity on high B-value images.

$4 \quad$ There was obviously focal low signal on the ADC image, and obviously high signal on the

score high B-value image. The maximum diameter of the axial plane was $<1.5 \mathrm{~cm}$.

$5 \quad$ Imaging findings were the same as 4 points, but the maximum diameter was $\geq 1.5 \mathrm{~cm}$, or score there was definite extension or invasion out of the prostate.

Table 5

PI-RADS v2 DCE score

\section{DCE standards of grading}

DCE (1)No reinforcement in the early stage.

negative

(2) Enhanced diffusion, with no corresponding focal expression on TWI or DWI.

(3) Corresponding lesions on DWI showed features of prostatic hyperplasia with focal enhancement.

Negative DCE is defined as having one of the three.

DCE Focal, prior to or concurrent with the enhancement of adjacent normal prostate tissue, positive consistent with the corresponding suspicious lesions of TWI and/or DWI.

Table 6

The central zone of PI-RADS V2

score

\begin{tabular}{|llll|}
\hline T2WI & DWI & DCE & Score \\
\hline 1 & Any & Any & 1 \\
\hline 2 & Any & Any & 2 \\
3 & $\leq 4$ & Any & 3 \\
\hline 3 & 5 & Any & 4 \\
\hline 4 & Any & Any & 4 \\
\hline 5 & Any & Any & 5 \\
\hline
\end{tabular}

Page $7 / 32$ 
Table 7

the PI-RADS V2 score of

peripheral zone

\begin{tabular}{|llll|}
\hline DWI & T2WI & DCE & Score \\
\hline 1 & Any & Any & 1 \\
2 & Any & Any & 2 \\
3 & Any & $(-)$ & 3 \\
3 & Any & $(+)$ & 4 \\
4 & Any & Any & 4 \\
5 & Any & Any & 5 \\
\hline
\end{tabular}

\subsubsection{Radiomics image texture analysis}

All ADC images were imported into the ONIS 2.5 Free Edition software in DICOM format. The images containing the lesion area were selected, exported, and saved as uncompressed DICOM files. ImageJ software was used to determine each lesion's full-layer volume and location for eligible patients according to T2WI and DWI sequences. Each lesion was manually delineated based on ADC images, and the characteristic texture parameters of full-layer lesion volume were derived. The 10th percentile (P10th), 25th percentile (P10th), 75th percentile (P75th), 90th percentile (P90th), mean ADC, median ADC, kurtosis, skewness, and MRI lesion volume were obtained. This is shown in Fig. 1.

\subsubsection{Selection of texture feature parameters}

Feature selection is an important part of statistical analysis and inference of data. In the actual modeling process, we need to identify independent variables with the strong explanatory ability to the dependent variables from the data to improve model prediction accuracy; that is, the quality of feature selection results directly affects the accuracy and quality of the model. We also used the LASSO regression to select the texture features of Mp-MRI in radiomics.

R software (version:4.0.4) lesions of export volume texture characteristic parameters of standardizing by package DMwR. The LASSO regression was used to analyze the characteristic parameters of dimensionreduction. The one thousand one hundred percent cross-validation method was used to select the optimal lambda values and then select the most representative characteristic values, thereby reducing the model complexity using the packages pROC and glmnet. Then, $70 \%$ of cases were randomly selected from the $\mathrm{PCa}$ and $\mathrm{BPH}$ groups as the training set, and the remaining $30 \%$ were the testing set. Normality testing, homogeneity testing of variance, and the t-test were performed on the training set. The Mann-Whitney 
test was performed on those who did not meet the t-test to obtain the latest training set. Finally, logistic regression was used to construct a PI-RADS 4/5 prediction model based on radiomics texture features to identify benign and malignant prostate lesions compared to the SVM and AdaBoost regressions.

\subsection{Model evaluation indexes}

\subsubsection{Prediction accuracy}

When a classification model is constructed, a confusion matrix is usually used to test the validity and practicability of the model. The confusion matrix is shown in Table 8. True positives (TP) indicate the correct prediction of carcinoma. False negatives (FN) represent incorrect predictions for cancer. False positives (FP) represent patients without cancer who were erroneously predicted to have cancer. True negatives (TN) did not have cancer and were correctly predicted to be as such. In a general data set, the classifier's performance can be evaluated according to the prediction accuracy, reflecting the classifier's ability to classify the whole sample.

Table 8

The confusion matrix

\begin{tabular}{|llll|}
\hline & \multicolumn{3}{l|}{ Prediction } \\
\cline { 2 - 4 } & & 1 & 0 \\
\hline Actual & 1 & TP & FN \\
\cline { 2 - 4 } & 0 & FP & TN \\
\hline Note: TP:True positives; FN:False negatives; FP:False positives; TN:True negatives \\
\hline
\end{tabular}

\subsubsection{ROC curve analysis}

When the output variable of the classifier is a probability value, an ROC curve is required. In the ROC curve, the abscissa is the FP rate or 1-specificity, and the ordinate is the TP rate or sensitivity. TPR represents the proportion of positive samples that are correctly classified, and FPR represents the proportion of negative samples that are misclassified. The formula is as follows:

TPR Sensitivity of $=$ TP $/+$ FN (TP) (1)

FPR $=1-$ Specificity $=$ FP $/+$ TN $(F P)(2)$

The ROC curve of the two-dimensional plane is as follows (Fig. 2):

The closer the ROC curve is to the upper left corner $(0,1)$, the better the classifier's performance. Although ROC curves can intuitively represent the performance of classifiers, specific values are sometimes needed to measure the quality of classification models. Therefore, the AUC of the ROC curve is often used to 
evaluate the regression's performance; AUC is the Riemann sum of the areas of differential trapezoids. The performance criteria of the model evaluated by AUC value are as follows: difference, $0.50-0.59$; general, 0.60-0.69; Good, 0.70-0.79; very good, 0.80-0.89; and excellent, 0.90-1.0.

\subsubsection{Nomogram}

We used the package rms in R software to draw a nomogram and its calibration curve. A nomogram combines several indicators based on multivariate regression analysis and plots them on the same plane with scale lines at a specific scale to demonstrate the relationships among variables in the model. Nomograms are widely used in medical research and clinical practice because they transform the complex regression equation into a visual image, making the results of the prediction model more readable and convenient for physicians directly to evaluate patients and their conditions and other characteristics.

\section{Results}

\subsection{R-logistic classification model based on texture features}

\subsubsection{Texture feature selection}

The data were standardized to eliminate errors caused by dimensionality difference, self-variation, or large numerical differences of each characteristic variable in the regression analysis. Then, the LASSO regression was used to reduce the dimensionality of ten texture feature parameters, and the minimum standard was used for ten cross-validations to select the optimal parameters of the model. The relationship curve between the minimum variance and $\log (\lambda)$ was made, and a vertical line was drawn at the optimal point (Fig. 3). The optimal parameter $\lambda$ in the LASSO model was set to 4 . According to the $\log (\lambda)$ sequence, the coefficient profile was drawn (Fig. 3), which is the LASSO coefficient profile of ten characteristic parameters. In the $\lambda$ graph, the vertical coordinate of the weight coefficient $\lambda$ curve was closer to 0 , indicating higher feature similarity. Vertical lines were drawn using ten cross-validations of the selected values, where the optimal lambda results in four features with non-zero coefficients. In conclusion, four important texture feature parameters can be obtained from MP-MRI-based ADC images: median ADC value, kurtosis, skewness, and MRI lesion volume.

\subsubsection{Construction and performance comparison of three ML classification models based on texture features}

The model building and model solving process of this study were all realized in $\mathrm{R}$, version 4.0.4. We used the essential texture features after LASSO dimension-reduction to build the model and established an ML model of logistic regression, SVM, and AdaBoost to predict the PI-RADS 4/5 benign and malignant nodule 
classifier. The PCa and BPH groups were randomly selected in a 7:3 ratio and divided into training and testing sets. The training set was used to train and construct the ML model, and the testing set was used to verify the model.

The four characteristic values identified above are as follows: median ADC, kurtosis, skewness, and MRI lesion volume were the input variables of the classification model, and whether prostate cancer $(0$ or 1$)$ was the output variable of the model. Logistic regression, SVM, and AdaBoost regression were used to construct the R-logistic, R-SVM, and R-AdaBoost classification models based on texture features. ROC curve analysis was used to compare the model performance of the three ML classification models, and the classification model with the largest area under the ROC curve was selected. The ROC curve analysis of each model was as follows. We selected the appropriate classifier by comparing the sensitivity, specificity, accuracy, and AUC values of the model.

(1) ROC curve analysis of R-logistic classification model

Logistic regression was carried out by combining $\mathrm{R}$ software and essential texture feature parameters based on ADC image feature selection. ROC curve analysis was conducted, as shown in Fig. 4. The AUC values of the ROC curves of the training and the testing sets were 0.936 and 0.838 . The sensitivity was 0.948 and 0.962 . The specificities were 0.571 and 0.600 , respectively. The accuracies were 0.875 and 0.903 , respectively, suggesting that the model had a good classification performance.

(2) ROC curve analysis of the R-SVM classification model

The SVM model was constructed by combining R software and essential texture feature parameters based on ADC image feature selection. ROC curve analysis was conducted (Fig. 5). The AUC values of the ROC curves of the training and the validation set were 0.845 and 0.800 , respectively. The sensitivities were 0.967 and 0.923 , the specificities were 0.623 and 0.600 , and the accuracies were 0.903 and 0.871 , respectively, suggesting that the model had a good classification performance.

(3) ROC curve analysis of R-AdaBoost classification model

The AdaBoost model was constructed by combining $\mathrm{R}$ language software and important texture parameter features based on $A D C$ image feature selection, and ROC curve analysis was conducted, respectively, as shown in Fig. 6 . The AUC values of the ROC curves of the training set and the testing set are 0.733 and 0.800 , the sensitivity is 0.966 and 0.923 , the specificity is 0.500 and 0.600 , and the accuracy is 0.875 and 0.871 , respectively. The model had a good classification performance.

The ROC curve analysis of these three classification models showed that the sensitivity, specificity, and accuracy of the testing group of the R-logistic classification model were $0.962,0.600$, and 0.903 , 
respectively. The sensitivity, specificity, and accuracy of the testing groups of the R-SVM classification model and the R-AdaBoost classification model were 0.923 and $0.923,0.600$ and 0.600 , and 0.871 and 0.871 , respectively (Table 9 ). The sensitivity and specificity of the training and testing sets of the three $\mathrm{ML}$ classification models showed slight differences; therefore, the AUC value was used as the evaluation index to judge the performance of the classification model. The AUC of the training and testing sets of the R-logistic classification model were 0.936 and 0.838 , respectively, which were higher than that of RSVM and R-AdaBoost classification models, suggesting that the R-logistic classification model had better classification performance. Therefore, the R-logistic classification model based on texture features was used as the classification model to differentiate benign from malignant prostate lesions in PI-RADS 4/5 score.

Table 9

Performance comparison of three kinds of machine learning

\begin{tabular}{|llllll|}
\hline Model & dataset & Sensitivity & specificity & Accuracy & AUC \\
\hline R-Logistic & Training set & 0.948 & 0.571 & 0.875 & 0.936 \\
\cline { 2 - 6 } & Testing set & 0.962 & 0.600 & 0.903 & 0.838 \\
\hline \multirow{2}{*}{ R-SVM } & Training set & 0.967 & 0.623 & 0.903 & 0.845 \\
\cline { 2 - 6 } & Testing set & 0.923 & 0.600 & 0.871 & 0.800 \\
\hline R-AdaBoost & Training set & 0.966 & 0.500 & 0.875 & 0.733 \\
\cline { 2 - 6 } & Testing set & 0.923 & 0.600 & 0.871 & 0.800 \\
\hline
\end{tabular}

\subsection{C-Logistic classification model based on clinical indicators}

\subsubsection{Treatment of clinical indicators}

The clinical indicators Age, PSA, fPSA, WBC, FIB, ALB, NLR, LDL-C, HDL-C, and TG were divided into PCa and $\mathrm{BPH}$ groups according to the pathological classification, and the t-test and homogeneity of variance test were performed for the clinical indicators. The Mann-Whitney test was used for data that were not normally distributed to determine any difference in data between the PCa and the BPH groups.

\subsubsection{Difference analysis of clinical indicators}

SPSS25.0 software was used to compare the clinical indicators in the PCa and the BPH groups. The statistical significance of each indicator is shown in Table 10. As can be seen from Table 10, the P-values 
of Age, PSA, fPSA, WBC, FIB, ALB, NLR, LDL-C, HDL-C, and TG were all $P<0.05$, suggesting that the differences in these indicators were statistically significant.

Table 10

Statistical test results of clinical indicators in PCA group and BPH group

\begin{tabular}{|c|c|c|c|c|}
\hline & PCa group & BPH group & t/U-value & P-value \\
\hline Sample & 83 & 20 & - & - \\
\hline Age & $71.39 \pm 8.624$ & $65.9 \pm 9.819$ & -2.567 & 0.012 \\
\hline BMI & $24.405 \pm 2.584$ & $23.435 \pm 3.836$ & -0.104 & 0.917 \\
\hline PSA & $25.989 \pm 17.740$ & $9.325 \pm 6.373$ & -4.944 & $<0.001$ \\
\hline fPSA & $7.071 \pm 12.817$ & $1.182 \pm 0.953$ & -3.473 & $<0.001$ \\
\hline \multicolumn{5}{|c|}{ Systemic Inflammatory Index } \\
\hline WBC & $6.793 \pm 1.830$ & $5.831 \pm 1.041$ & -2.189 & 0.029 \\
\hline Fib & $3.133 \pm 0.766$ & $4.44 \pm 1.585$ & -3.927 & $<0.001$ \\
\hline ALB & $38.166 \pm 3.554$ & $42.125 \pm 4.287$ & 4.293 & $<0.001$ \\
\hline NLR & $4.077 \pm 2.255$ & $2.645 \pm 1.249$ & -2.526 & 0.012 \\
\hline CPR & $6.755 \pm 8.676$ & $4.025 \pm 4.690$ & -0.984 & 0.325 \\
\hline PLT & $184.76 \pm 49.599$ & $224.95 \pm 84.756$ & -1.643 & 0.100 \\
\hline \multicolumn{5}{|c|}{ Metabolism Index } \\
\hline LDL-C & $2.796 \pm 0.789$ & $2.093 \pm 0.545$ & -3.770 & $<0.001$ \\
\hline HDL-C & $1.229 \pm 0.356$ & $1.003 \pm 0.159$ & -2.771 & 0.007 \\
\hline TG & $1.441 \pm 0.695$ & $1.047 \pm 0.408$ & -2.431 & 0.017 \\
\hline
\end{tabular}

\subsubsection{ROC curve analysis of clinical indicators}

R software was used to analyze the ROC curves of clinical indicators, as shown in Fig. 7. As can be seen from the figure, the AUC values of ROC curves of PSA, FIB, ALB, fPSA, NLR, WBC, LDL-C, HDL-C and TG were $0.857,0.784,0.761,0.751,0.683,0.658,0.778,0.740$, and 0.733 , respectively. All showed high efficacy in differentiating benign and malignant prostate lesions. 


\subsubsection{C-logistic classification model performance based on clinical indicators}

To reduce the complexity of the model, using difference analysis of clinical indicators and ROC curve analysis, the top three clinical indicators with the largest AUC values were selected to construct the Clogistic classification model; $70 \%$ of the samples of $\mathrm{PCa}$ and $\mathrm{BPH}$ groups were selected as the training set, PSA, FIB, and LDL-C were taken as the input variables of the model, and corresponding cancer and non-cancer were taken as the output variables. The logistic classification model was established by combining R language software and clinical indicators, and ROC curve analysis was conducted (Fig. 8). The AUC value of the ROC curves of the training set and the testing set were 0.860 and 0.738 , respectively. The sensitivities were 0.967 and 0.855 , the specificities were 0.857 and 0.800 , and the accuracies were 0.944 and 0.871 , respectively, suggesting that the model had a good classification performance.

\subsection{Comparative analysis of R-logistic model and C-logistic model}

\subsubsection{Logistic regression equation of characteristic texture parameters and clinical indicators}

Logistic regression was used to construct logistic regression equations for texture feature data and clinical index data based on imaging omics. The parameters and their coefficients are shown in Table 11. The R-logistic regression equation based on the texture features of imagomics was $Y \_$Radiomics $=$ 9.396-7.464 * Median ADC -0.584 * Kurtosis + 0.627 *Skewness + 0.576 * MRI lesion volume. The formula of $\mathrm{C}$-logistic regression equation based on clinical indicators was as follows: $Y_{-}$clinical $=-2.608$ +0.324 * PSA $-3.045 *$ FIB +4.147 * LDL-C. 
Table 11

Multivariate logistic regression model for predicting prostate cancer risk

\begin{tabular}{|lllll|}
\hline Model & Intercept and variable & \multicolumn{3}{l|}{ Prediction model } \\
\hline R-logistic & & $\beta$ & Odds ratio $(95 \% \mathrm{Cl})$ & P-value \\
\cline { 2 - 5 } & Intercept & 9.396 & $10.212(9.239-15.238)$ & $<0.001$ \\
\cline { 2 - 5 } & median ADC & -7.464 & $1.101(0.501-2.02)$ & $<0.001$ \\
\cline { 2 - 5 } & kurtosis & -0.584 & $0.558(0.249-1.192)$ & 0.115 \\
\cline { 2 - 5 } & skewness & 0.627 & $1.872(0.864-4.961)$ & 0.151 \\
\cline { 2 - 5 } & MRI lesion volume & 0.576 & $1.780(0.744-5.131)$ & 0.229 \\
\hline C-logistic & Intercept & -2.608 & $0.023(0.015-0.049)$ & 0.592 \\
\cline { 2 - 5 } & PSA & 0.324 & $1.382(1.137-1.987)$ & 0.016 \\
\cline { 2 - 5 } & Fib & -3.045 & $0.048(0.01-0.413)$ & 0.051 \\
\cline { 2 - 5 } & LDL-C & 4.147 & $63.268(4.726-7178.178)$ & 0.017 \\
\hline Note: $\beta$ is the regression coefficient; Cl confidence interval. & \\
\hline
\end{tabular}

The t-test was carried out according to the logistic regression equation, and the corresponding results are shown in Table 12. The Y_Radiomics in the training group PCa with R-logistic regression equation was $2.738 \pm 1.538$, and the $Y \_$Radiomics in the training group BPH was $-0.270 \pm 2.519$. The P-value between the two groups was less than 0.001 . The training set of the C-logistic regression equation was $4.364 \pm$ 8.403 in the PCa group and $-4.265 \pm 5.441$ in the BPH group. The P-value between groups was less than 0.001 . In other words, both sets of equations can be used in studies distinguishing benign from malignant lesions of the prostate in PI-RADS 4/5 score.

Table 12

T-test of R-logistic and C-logistic regression equations

\begin{tabular}{|lllll|}
\hline Model & PCa group & BPH group & t-value & P-value \\
\hline Sample $(n)$ & 58 & 14 & - & - \\
\hline R-logistic Model & $2.738 \pm 1.538$ & $-0.270 \pm 2.519$ & -5.733 & $<0.001$ \\
\hline C-logistic Model & $4.364 \pm 8.403$ & $-4.265 \pm 5.441$ & -3.651 & $<0.001$ \\
\hline
\end{tabular}

In summary, ROC curve analysis results of the R-logistic classification model based on texture features and C-logistic classification model based on clinical indicators are shown in Table 13 and Fig. 9. The AUC values of training and testing sets of the R-logistic classification model were 0.936 and 0.838 , respectively, which were higher than that of the C-logistic classification model based on clinical indicators. In other words, when differentiating benign and malignant prostate lesions to establish an $\mathrm{ML}$ classifier model, Mp-MRI radiomics texture features were superior to clinical indicators. When the Yoden 
index was at its maximum, the cutoff value for identifying benign and malignant prostate lesions in the PI-RADS 4/5 score was 1.216; that is, when Y_Radiomics was greater than 1.216, the model would predict prostate cancer, with sensitivity and specificity of $87.9 \%$ and $79.0 \%$, respectively.

Table 13

Performance comparison of R-logistic and C-logistic classification models

\begin{tabular}{|llllll|}
\hline Model & & Sensitivity & specificity & Accuracy & AUC \\
\hline R-logistic & training set & 0.948 & 0.571 & 0.875 & 0.936 \\
\cline { 2 - 6 } & testing set & 0.962 & 0.600 & 0.903 & 0.838 \\
\hline \multirow{2}{*}{ C-logistic } & training set & 0.967 & 0.857 & 0.944 & 0.860 \\
\cline { 2 - 6 } & testing set & 0.855 & 0.800 & 0.871 & 0.738 \\
\hline
\end{tabular}

\subsubsection{Further analysis of R-logistic classification model}

A nomogram for predicting the risk of benign and malignant prostatic lesions was drawn based on Y_Radiomics (Fig. 10). The nomogram shows a patient's total score of imaging texture features for predicting the risk of benign and malignant prostatic lesions. Combined with its calibration curve (Fig. 11), the performance demonstrated good predictive ability. The X-axis, Y-axis, oblique dotted line, and solid line in the calibration curve represent the meanings of the predicted risk of benign and malignant prostate lesions, the probability of actual diagnosis, the perfect prediction of the ideal model, and the performance of the nomogram, respectively. According to the calibration curve, the nomogram of Y_Radiomics showed a good predictive performance for benign and malignant prostate lesions. The nomogram chart that is closer to the diagonal dotted line has a better prediction.

\section{Discussion}

In recent years, the incidence of prostate cancer in China has shown a significant upward trend because early clinical symptoms of $\mathrm{PCa}$ and $\mathrm{BPH}$ are very similar; however, the treatment regimens are quite different. Prostate biopsy is the gold standard for diagnosing and assessing the risk of prostate cancer; however, it can cause complications because it is an invasive test. PSA is of great value in the screening of prostate cancer; however, it is nonspecific. 
Improving the early diagnosis rate of prostate cancer can improve survival. Many studies have tried to improve the diagnostic efficiency of prostate cancer using several clinical experimental indicators and diagnostic models. Wang et al. ${ }^{[20]}$ retrospectively analyzed patients who underwent prostate biopsy and evaluated age, PSA level, prostate volume, number of prostate biopsies, DRE results, and transrectal ultrasound for the presence of hypoechoic lesions. Logistic regression was used to create an equation to predict the likelihood of developing prostate cancer. Li et al. ${ }^{[21]}$ established a logistic regression model to provide the basis for a prostate needle biopsy, and found that DRE, TRUS, MRI, PSAD, and f/tPSA affected prostate biopsy. According to the logistic regression model of prostate cancer established by regression coefficient, prostate biopsy should be performed when the P-value is greater than 0.12 .

In the present study, we considered PSA, fPSA, systemic inflammatory indicators, and metabolic indicators of patients. Using the t-test and ROC curve analysis results, we found that the AUC value of PSA was 0.857 , which was higher than the systemic inflammatory indicators and metabolic indicators. We selected the best three indicators to establish a logistic regression model to differentiate benign and malignant prostate lesions to improve the accuracy and efficiency of the differentiation of benign and malignant prostate lesions and obtained Y_Clinical $=-2.608+0.324$ * PSA -3.045 * FIB + 4.147 * LDL-C. The AUC values of the training and testing sets were 0.860 and 0.738 , respectively.

Texture analysis (TA) in radiomics is a processing process that uses image processing software to extract texture characteristic parameters and obtain quantitative or qualitative descriptions of texture. TA has been applied in medicine. TA of CT, MRI, and PET-CT images can be used to obtain texture parameters related to disease, which is essential for detection and diagnosis of prostate cancer, GS score and clinical staging, prediction of recurrence, metastasis, and efficacy evaluation. Wibmer et al. ${ }^{[22]}$ explored Haralick TA of prostate MRI to evaluate prostate cancer diagnosis and pathological grading and found that the energy and entropy of texture feature extraction were significantly different for the diagnosis of prostate cancer and in tumors with $G S \leq 3+4$ and $\geq 4+3$. Xiong et al. ${ }^{[23]}$ explored the potential value of MRI texture analysis combined with prostate-related biomarkers for predicting highgrade prostate cancer (HGPCA). Logistic regression and ROC curve analysis of TA parameters from T2WI and DWI in the HGPCA group and non-high-grade prostate cancer group (NHGPCA) showed kurtosis, skewness, and entropy obtained from ADC mapping predicted HGPCA. Pan et al. ${ }^{[24]}$ discussed the value of TA in evaluating the grade of prostate cancer and found that texture features in tumor areas in T2WI were extracted and analyzed using texture software. The authors found that with increased GS, entropy increased and energy decreased, and the energy value of GS $=6$ groups was significantly higher than that of GS $=8$ and GS $=9-10$ groups. Daniel et al. ${ }^{[25]}$ used the texture feature (TF) method to investigate the value of dual-parameter MR in prostate cancer patients with and without neoadjuvant androgen removal and found that the sensitivity obtained using TF was equal or higher in the group without neoadjuvant androgen removal.

Many studies extracted the textural parameters of the region of interest from a single layer to obtain the TF of the lesion. Imaging studies in other tissues have demonstrated the importance of measuring 
textural parameters using an approach based on whole lesion volume and providing a complete representation of the entire tumor. This also facilitates the calculation of more advanced metrics to reflect the overall lesion texture and inhomogeneity ${ }^{[26,27]}$. In the present study, texture parameters were determined from all ADC images that included lesions covering the entire 4/5 of the PI-RADS V2 score. This analytical approach may better reveal intra-tumor heterogeneity and improve clinical evaluation.

$\mathrm{ML}$ is the core of artificial intelligence. It studies how to make computer-aided systems build models from data and use the established models to predict new inputs. Cuocolo et al. ${ }^{[28]}$ described a summary of the recent application of $\mathrm{ML}$ in radiology, emphasizing the application of $\mathrm{ML}$ and deep learning in prostate MRI. The application of ML in prostate cancer localization, diagnosis, local staging, and pre-treatment assessment, treatment, and prediction of biochemical recurrence was outlined, demonstrating the potential of the combination of ML and MRI to automate the classification of clinically significant prostate cancer in assisting clinical decision-making.

Based on R software, we performed classification, regression, clustering, dimension reduction, model selection, statistical analysis, and data preprocessing, which can quickly complete the entire process of machine learning ${ }^{[29]}$. By combining TA and ML, Stanford et al. ${ }^{[30]}$ determined whether an $\mathrm{ML}$ regression could use TA features extracted from unenhanced MR images to predict prostate cancer histopathology and concluded that the ML and TA combination method was a feasible tool for predicting histopathology of prostate cancer on two-parameter MR images. Alvarez-Jimenez et al. ${ }^{[31]}$ used the combination of TF with SVM, AdaBoost, and a random forest model to identify the regions where prostate cancer occurred. The authors highlighted the value of TA and $M L$ in the localization of prostate cancer. Wu et al. ${ }^{[32]}$ extracted texture and shape features from the apparent diffusion coefficient images, established a model using logistic regression and SVM to diagnose peripheral band prostate cancer and concluded that LR and SVM models were accurate for diagnosing peripheral band prostate cancer.

In the present study, we considered patients whose Mp-MRI examination indicated PI-RADS V2 4/5 score and used ImageJ and other software to extract the TF of all lesions. R software was used to conduct data normalization for these TF parameters, and the LASSO regression was performed to reduce the dimensions of the parameters. The four most essential feature parameters were obtained: median ADC, kurtosis, skewness, and MRI lesion volume. These texture parameters were combined using SVM, Logistic regression, and an AdaBoost ML model to establish a classifier model for prostate cancer. The training and testing sets were obtained by random sampling according to a 7:3 ratio. We found that the AUC values and accuracy of SVM, logistic regression, and AdaBoost models' training and testing sets were high, and there was a slight difference in AUC values between the training and testing sets, suggesting that these three classifier models have substantial application value. The AUC value of the ROC curve of the Logistic regression testing set was 0.838 , which was higher than the prediction model of benign and malignant prostate lesions established by SVM and AdaBoost, and similar to the results of other researchers, suggesting the essential clinical value of Logistic regression in the differentiation of benign from malignant prostate lesions. 
Magnetic resonance examination of the prostate can save patients from the trauma and potential complications caused by prostate biopsy; however, magnetic resonance detection of clinically significant prostate cancer has limitations, especially since the accuracy of diagnosis varies significantly between individual radiologists ${ }^{[3]}$. Imaging omics TA uses advanced image processing technology to extract many feature parameters from image images, and its potential to improve diagnostic accuracy has been confirmed by several studies ${ }^{[34]}$. In the present study, Logistic regression was applied to construct a classification model for prostate cancer based on the texture features of imaging omics. In this case, the corresponding R-logistic classification model was Y_radiomics $=9.396-7.464$ * Median ADC -0.584 * Kurtosis + 0.627 * Skewness + 0.576 * MRI lesion volume. The AUC value of the ROC curve of the Rlogistic training set was 0.936 , which was better than that of the C-logistic classification model. When the Youden index was at its maximum, the cutoff point value for differentiating benign from malignant prostatic lesions was 1.216 , and its sensitivity and specificity were $87.9 \%$ and $79.0 \%$, respectively. Because these four variables in the PI-RADS 4/5 medium prostate lesions model can be easily obtained clinically, we believe that this equation can predict prostate cancer in daily clinical practice. Nomograms are widely used in oncology to predict outcomes. Nomograms rely on the advantages of a friendly digital

interface and higher accuracy to help better clinical decision-making ${ }^{[35]}$. Our nomogram was based on $Y$ (radiomics) to differentiate benign from malignant prostate lesions, and its calibration curve showed good predictive power.

In summary, based on patients with a 4/5 PI-RADS V2 score, we extracted TP in Mp-MRI combined with the ML models R-SVM, R-logistic regression, and an R-AdaBoost classifier to establish a prediction model discriminating between benign and malignant prostate lesions. The AUC of the R-logistic regression classifier was the highest and better than that of C-logistic regression established by clinical indicators, suggesting that the application value of PI-RADS $4 / 5$ score in predicting benign and malignant prostate lesions was good.

There are some limitations to this study. First, the sample size was relatively small, and all studies were retrospective; therefore, a large sample size expansion is needed to conduct prospective studies in the future. Second, the samples in this study were from a single center, and there is a lack of independent verification data set. In the future, a multi-center study will be used to validate the results of the present study. Third, this study manually delineated the ROI, which is inefficient and subjective to some extent and is not conducive to big data processing. In the future, it may be possible to use computer-aided systems to identify and segment lesions automatically.

\section{Conclusion}

The machine learning classifier model is established based on the texture features of radiomics. It has a good classification performance in identifying benign and malignant nodules of the prostate in PI-RADS $4 / 5$. This has certain potential and clinical value for patients with prostate cancer to adopt different treatment methods and prognosis. 


\section{Abbreviations}

\begin{tabular}{|ll|}
\hline Mp-MRI & Multiparametric MRI \\
\hline PCa & prostate cancer \\
\hline Lasso & Least absolute shrinkage and selection operator \\
\hline PI-RADS & Prostate Imaging Report and Data System \\
\hline SVM & Support vector machine \\
\hline LR & Logistic Regression \\
\hline Ada & Adaptive Boosting \\
\hline ROC & Receiver Operating Characteristic \\
\hline AUC & Area Under Curve \\
\hline PSA & prostate specific antigen \\
\hline BPH & Benign Prostate Hyperplasia \\
\hline GS & Gleason score \\
\hline Al & Artificial Intelligence \\
\hline ML & Machine learning \\
\hline LDA & linear discriminant analysis \\
\hline DT & Decision Tree \\
\hline NT & Neural networks \\
\hline ADC & Apparent diffusion coefficient \\
\hline DWI & diffusion-weighted imaging \\
\hline GS & Gleason score \\
\hline
\end{tabular}

\section{Declarations}

Ethics approval and consent to participate: All procedures performed in studies involving human participants were in accordance with the ethical standards of the institutional and national research committee. The Ethics Committee of the First Affiliated Hospital of Soochow University approved the study.

Consent for publication: yes 
Availability of data and materials:The datasets used and analysed during the current study are available from the corresponding author on reasonable request.

Competing interests: Author Lu Ma declares that he has no conflict of interest. Author Qi Zhou declares that he has no conflict of interest. Author Huming Yin declares that he has no conflict of interest. Author Xiaojie Ang declares that he has no conflict of interest. Author Yu Li declares that he has no conflict of interest. Author Gansheng Xie declares that he has no conflict of interest. Author Gang Li declares that he has no conflict of interest.

Funding: This study was not funded.

Authors' contributions: (I) Conception and design: Lu Ma; (II) Administrative support: Gang Li; (III) Provision of study materials or patients:Lu Ma, Qi Zhou, Huming Yin, Xiaojie Ang,Yu Li,Gansheng Xie; (IV) Collection and assembly of data: Lu Ma*, Qi Zhou*; (V) Data analysis and interpretation: Lu Ma, Xiaojie Ang; (VI) Manuscript writing: All authors ;(VII) Final approval of manuscript: All authors. ${ }^{*}$ These authors contributed equally to this work.

Acknowledgements: Not applicable

\section{References}

[1] CULP M B, SOERJOMATARAM I, EFSTATHIOU J A, et al. Recent Global Patterns in Prostate Cancer Incidence and Mortality Rates [J]. Eur Urol, 2020, 77(1): 38-52.

[2] SCHATTEN H. Brief Overview of Prostate Cancer Statistics, Grading, Diagnosis and Treatment Strategies [J]. Adv Exp Med Biol, 2018, 1095(1-14).

[3] SIEGEL R L, MILLER K D, JEMAL A. Cancer statistics, 2018 [J]. CA Cancer J Clin, 2018, 68(1): 7-30.

[4] CHEN W, ZHENG R, BAADE P D, et al. Cancer statistics in China, 2015 [J]. CA Cancer J Clin, 2016, 66(2): 115-132.

[5] GEORGE A K, TURKBEY B, VALAYIL S G, et al. A urologist's perspective on prostate cancer imaging: past, present, and future [J]. Abdom Radiol (NY), 2016, 41(5): 805-816.

[6] SUN Y, REYNOLDS H M, PARAMESWARAN B, et al. Multiparametric MRI and radiomics in prostate cancer: a review [J]. Australas Phys Eng Sci Med, 2019, 42(1): 3-25.

[7] MANFREDI M, MELE F, GARROU D, et al. Multiparametric prostate MRI: technical conduct, standardized report and clinical use [J]. Minerva Urol Nefrol, 2018, 70(1): 9-21.

[8] CHOI E R, LEE H Y, JEONG J Y, et al. Quantitative image variables reflect the intratumoral pathologic heterogeneity of lung adenocarcinoma [J]. Oncotarget, 2016, 7(41): 67302-67313. 
[9] LI M, CHEN T, ZHAO W, et al. Radiomics prediction model for the improved diagnosis of clinically significant prostate cancer on biparametric MRI [J]. Quant Imaging Med Surg, 2020, 10(2): 368-379.

[10] HAMM C A, BEETZ N L, SAVIC L J, et al. [Artificial intelligence and radiomics in MRI-based prostate diagnostics] [J]. Radiologe, 2020, 60(1): 48-55.

[11] HARMON S A, TUNCER S, SANFORD T, et al. Artificial intelligence at the intersection of pathology and radiology in prostate cancer [J]. Diagn Interv Radiol, 2019, 25(3): 183-188.

[12] PANTANOWITZ L, QUIROGA-GARZA G M, BIEN L, et al. An artificial intelligence algorithm for prostate cancer diagnosis in whole slide images of core needle biopsies: a blinded clinical validation and deployment study [J]. Lancet Digit Health, 2020, 2(8): e407-e416.

[13] STARK G F, HART G R, NARTOWT B J, et al. Predicting breast cancer risk using personal health data and machine learning models [J]. PLoS One, 2019, 14(12): e0226765.

[14] TREBESCHI S, DRAGO S G, BIRKBAK N J, et al. Predicting response to cancer immunotherapy using noninvasive radiomic biomarkers [J]. Ann Oncol, 2019, 30(6): 998-1004.

[15] HORVAT N, BATES D D B, PETKOVSKA I. Novel imaging techniques of rectal cancer: what do radiomics and radiogenomics have to offer? A literature review [J]. Abdom Radiol (NY), 2019, 44(11): 3764-3774.

[16] NAKAMOTO T, HAGA A, TAKAHASHI W. [An Introduction to Radiomics: Toward a New Era of Precision Medicine] [J]. Igaku Butsuri, 2018, 38(3): 129-134.

[17] MORIN A, FRITSCH L, MATHIEU J R, et al. Identification of CAD as an androgen receptor interactant and an early marker of prostate tumor recurrence [J]. Faseb j, 2012, 26(1): 460-467.

[18] SCHMUECKING M, BOLTZE C, GEYER H, et al. Dynamic MRI and CAD vs. choline MRS: where is the detection level for a lesion characterisation in prostate cancer? [J]. Int J Radiat Biol, 2009, 85(9): 814-824.

[19] WEINREB J C, BARENTSZ J O, CHOYKE P L, et al. PI-RADS Prostate Imaging - Reporting and Data System: 2015, Version 2 [J]. Eur Urol, 2016, 69(1): 16-40.

[20] WANG J C, HUAN S K, KUO J R, et al. A multivariable logistic regression equation to evaluate prostate cancer [J]. J Formos Med Assoc, 2011, 110(11): 695-700.

[21] LI Y, TANG Z, QI L, et al. [Analysis of influential factors for prostate biopsy and establishment of logistic regression model for \prostate cancer] [J]. Zhong Nan Da Xue Xue Bao Yi Xue Ban, 2015, 40(6): 651-656.

[22] WIBMER A, HRICAK H, GONDO T, et al. Haralick texture analysis of prostate MRI: utility for differentiating non-cancerous prostate from prostate cancer and differentiating prostate cancers with 
different Gleason score [J]. Eur Radiol, 2015, 25(10): 2840-2850.

[23] XIONG H, HE X, GUO D. Value of MRI texture analysis for predicting high-grade prostate cancer [J]. Clin Imaging, 2021, 72(168-174).

[24] PAN R, YANG X, SHU Z, et al. Application of texture analysis based on T2-weighted magnetic resonance images in discriminating Gleason score of prostate cancer [J]. J Xray Sci Technol, 2020, 28(6): 1207-1218.

[25] DANIEL M, KUESS P, ANDRZEJEWSKI P, et al. Impact of androgen deprivation therapy on apparent diffusion coefficient and T2W MRI for histogram and texture analysis with respect to focal radiotherapy of prostate cancer [J]. Strahlenther Onkol, 2019, 195(5): 402-411.

[26] YIP S S F, LIU Y, PARMAR C, et al. Associations between radiologist-defined semantic and automatically computed radiomic features in non-small cell lung cancer [J]. Sci Rep, 2017, 7(1): 3519.

[27] KIERANS A S, BENNETT G L, MUSSI T C, et al. Characterization of malignancy of adnexal lesions using $A D C$ entropy: comparison with mean $A D C$ and qualitative $D W I$ assessment [J]. J Magn Reson Imaging, 2013, 37(1): 164-171.

[28] CUOCOLO R, CIPULLO M B, STANZIONE A, et al. Machine learning applications in prostate cancer magnetic resonance imaging [J]. Eur Radiol Exp, 2019, 3(1): 35.

[29] CHEN X W, GAO J X. Big Data Bioinformatics [J]. Methods, 2016, 111(1-2).

[30] STANZIONE A, CUOCOLO R, COCOZZA S, et al. Detection of Extraprostatic Extension of Cancer on Biparametric MRI Combining Texture Analysis and Machine Learning: Preliminary Results [J]. Acad Radiol, 2019, 26(10): 1338-1344.

[31] ALVAREZ-JIMENEZ C, BARRERA C, MUNERA N, et al. Differentiating Cancerous and Non-cancerous Prostate Tissue Using Multi-scale Texture Analysis on MRI [J]. Annu Int Conf IEEE Eng Med Biol Soc, 2019, 2019(2695-2698).

[32] WU M, KRISHNA S, THORNHILL R E, et al. Transition zone prostate cancer: Logistic regression and machine-learning models of quantitative $A D C$, shape and texture features are highly accurate for diagnosis [J]. J Magn Reson Imaging, 2019, 50(3): 940-950.

[33] ROSENKRANTZ A B, AYOOLA A, HOFFMAN D, et al. The Learning Curve in Prostate MRI Interpretation: Self-Directed Learning Versus Continual Reader Feedback [J]. AJR Am J Roentgenol, 2017, 208(3): W92-w100.

[34] BOROFSKY S, GEORGE A K, GAUR S, et al. What Are We Missing? False-Negative Cancers at Multiparametric MR Imaging of the Prostate [J]. Radiology, 2018, 286(1): 186-195. 
[35] WEI L, CHAMPMAN S, LI X, et al. Beliefs about medicines and non-adherence in patients with stroke, diabetes mellitus and rheumatoid arthritis: a cross-sectional study in China [J]. BMJ Open, 2017, 7(10): e017293.

\section{Figures}
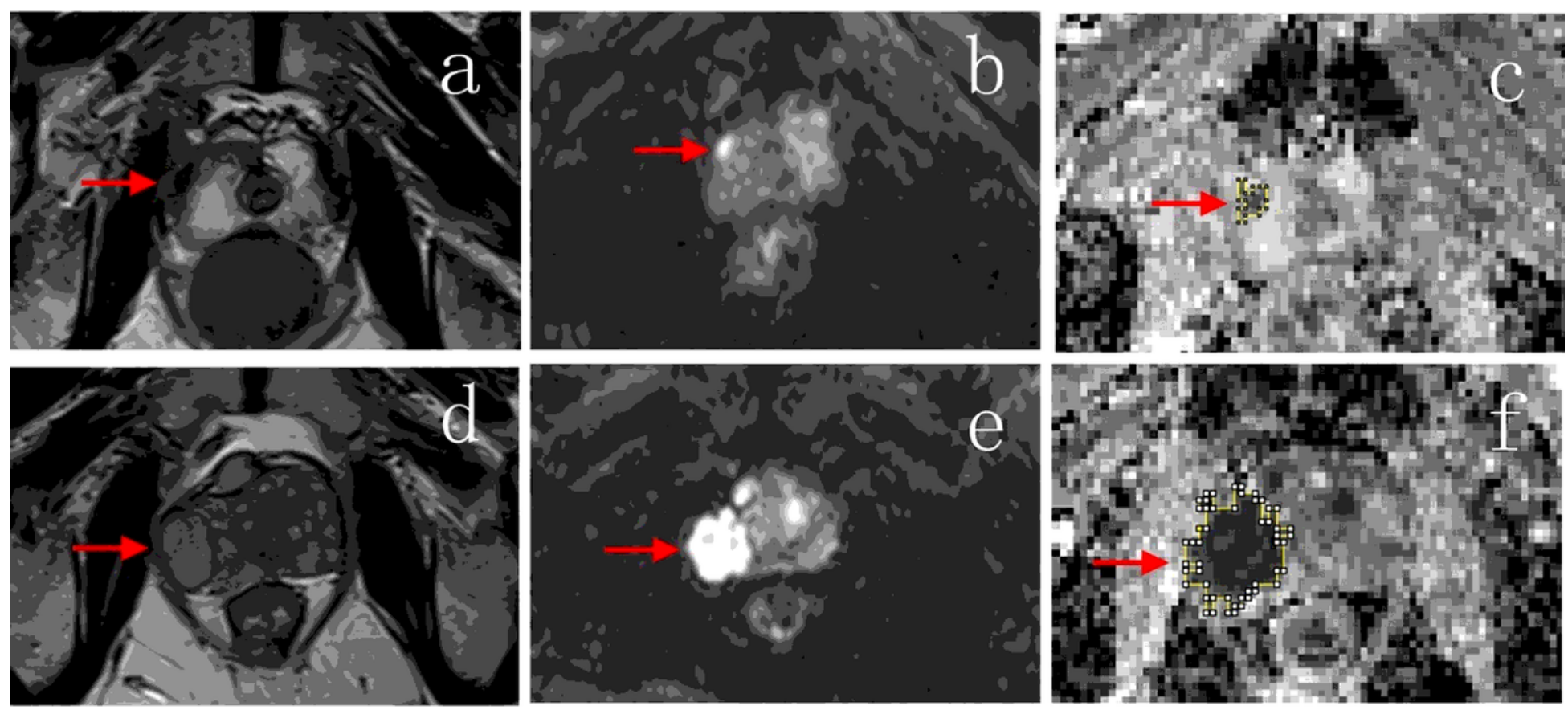

Figure 1

MRI images of PCa and BPH 


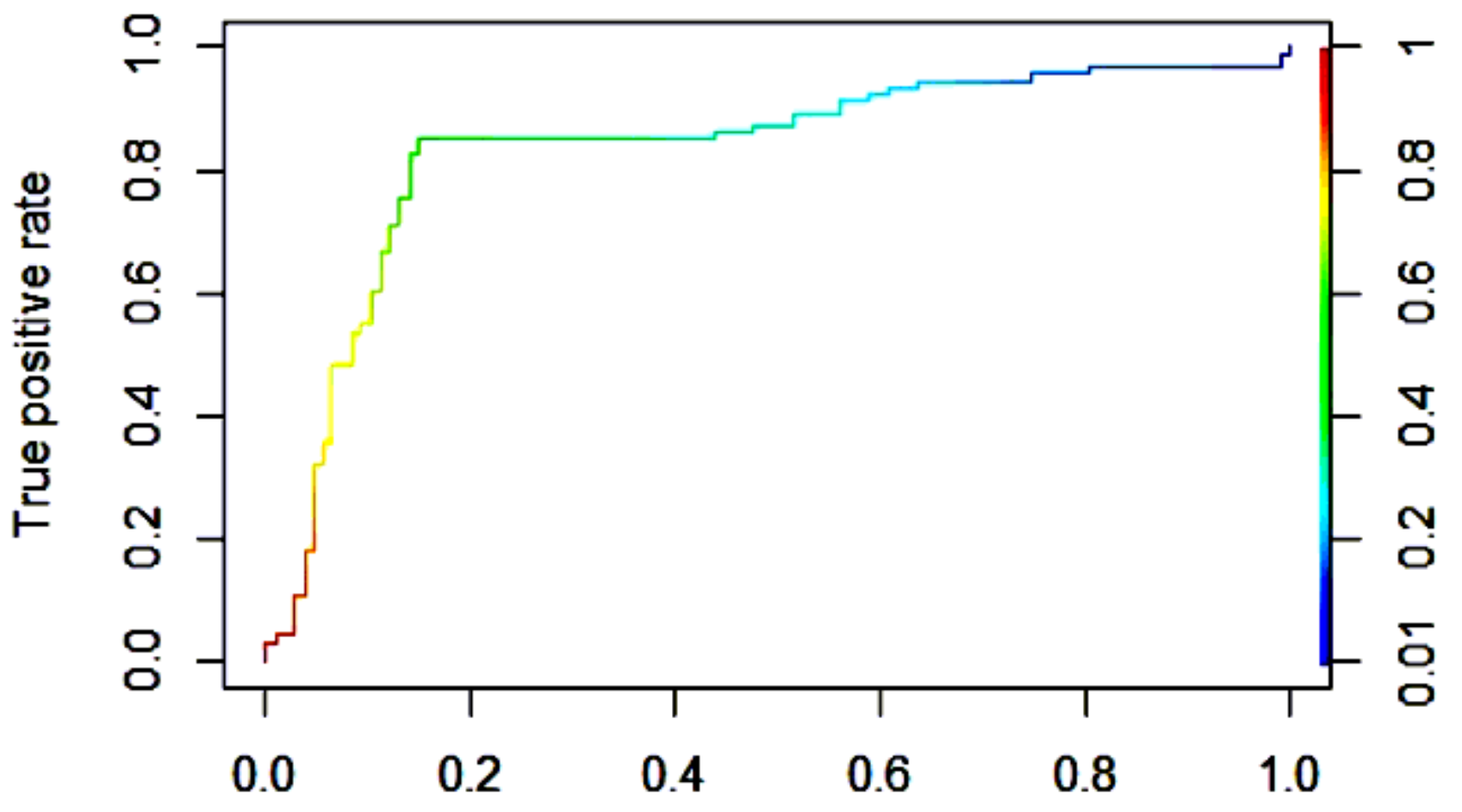

False positive rate

Figure 2

ROC curve
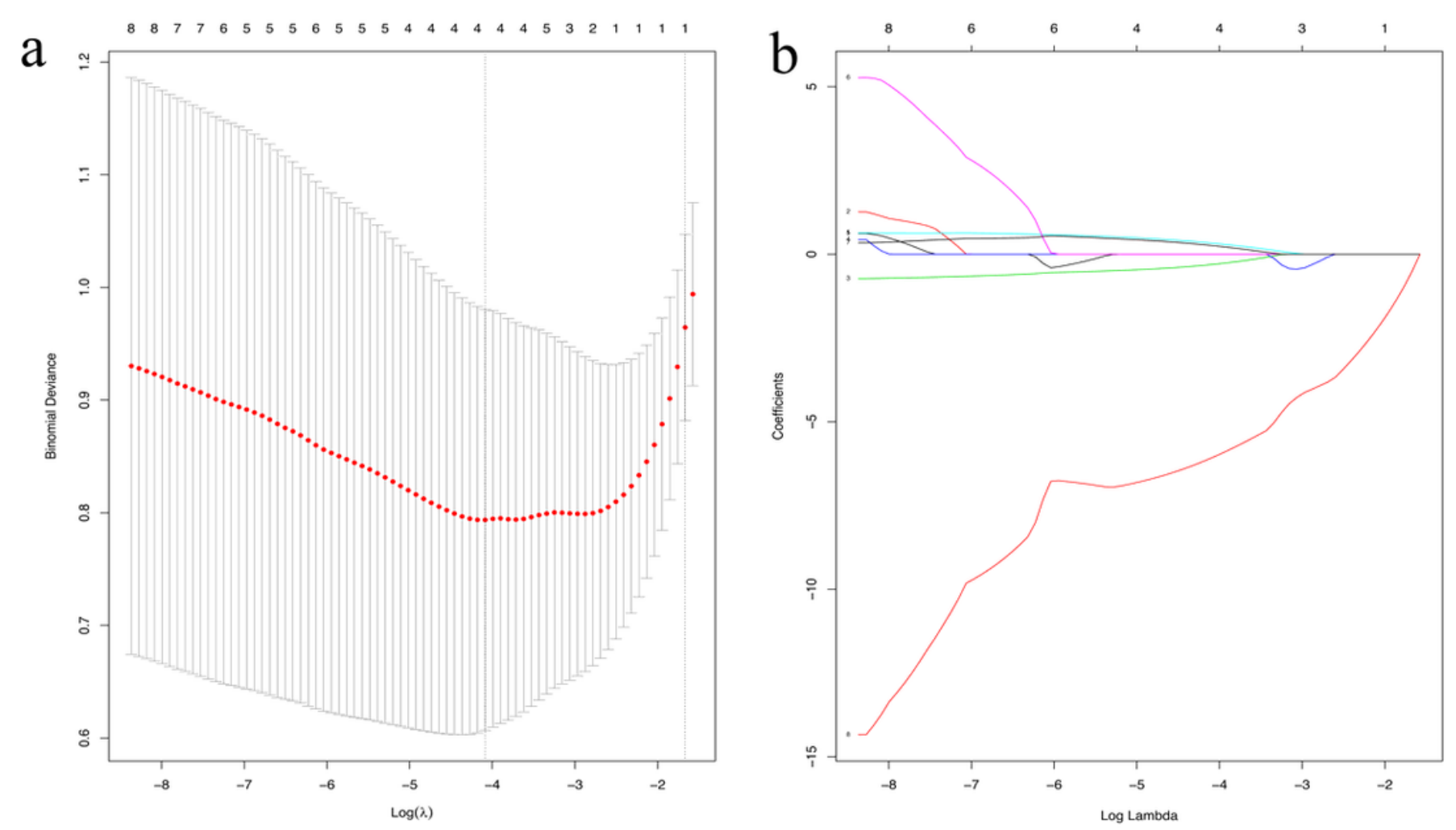


\section{Figure 3}

Binomial deviation curve of radiomics model with parameter $\lambda$. The vertical axis represents the binomial deviation. The horizontal axis represents the $\log (\lambda)$ value. The upper number represents the optimal value (the perpendicular dotted line), where the smallest binomial deviation is found for the quantitative model with the selected features. b Graph of radiomics characteristic parameters changing with $\lambda$.
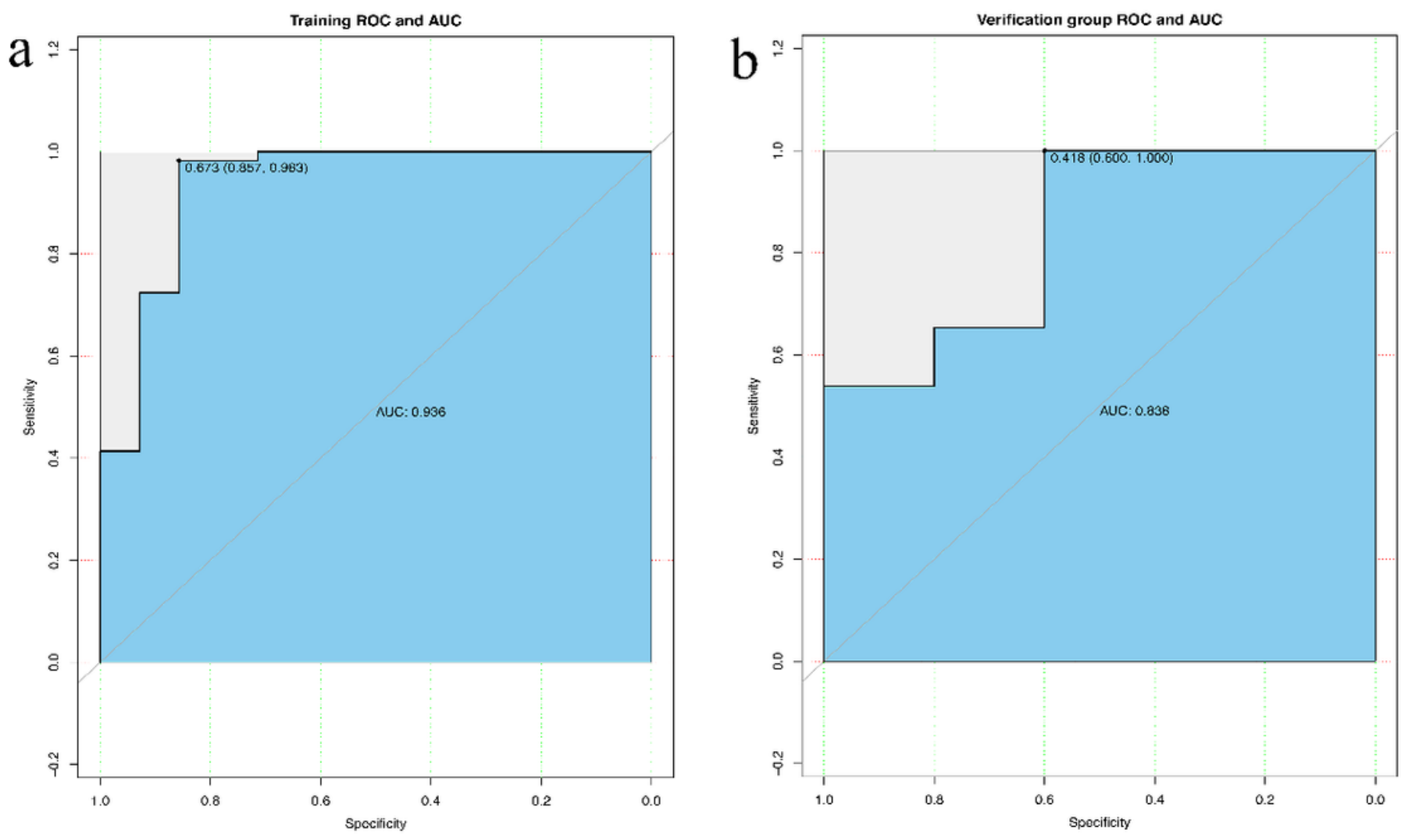

Figure 4

ROC curve analysis of training set (a) and verification set (b) in the logistic regression model 

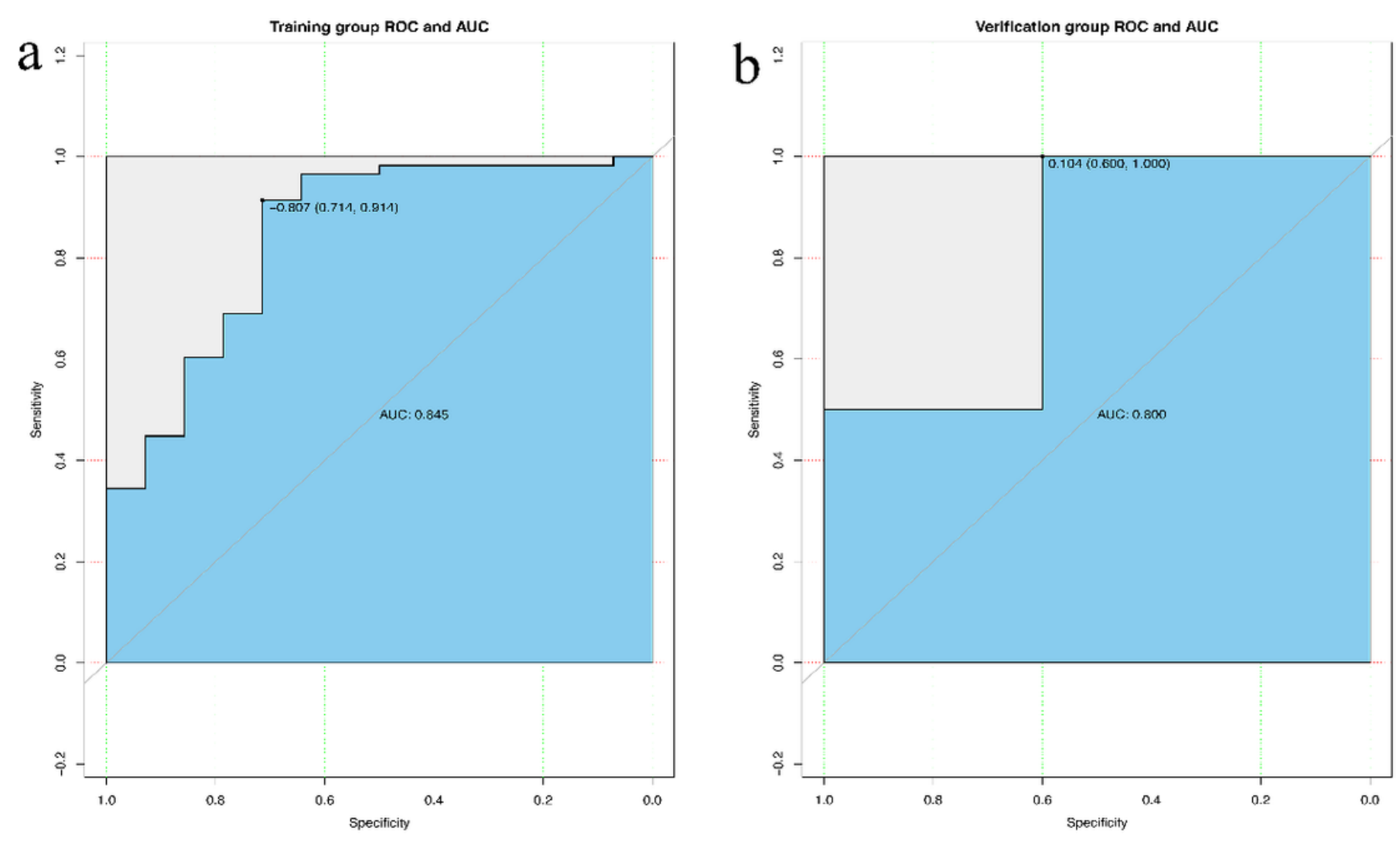

\section{Figure 5}

ROC curve analysis of training set (a) and verification set (b) in the SVM model
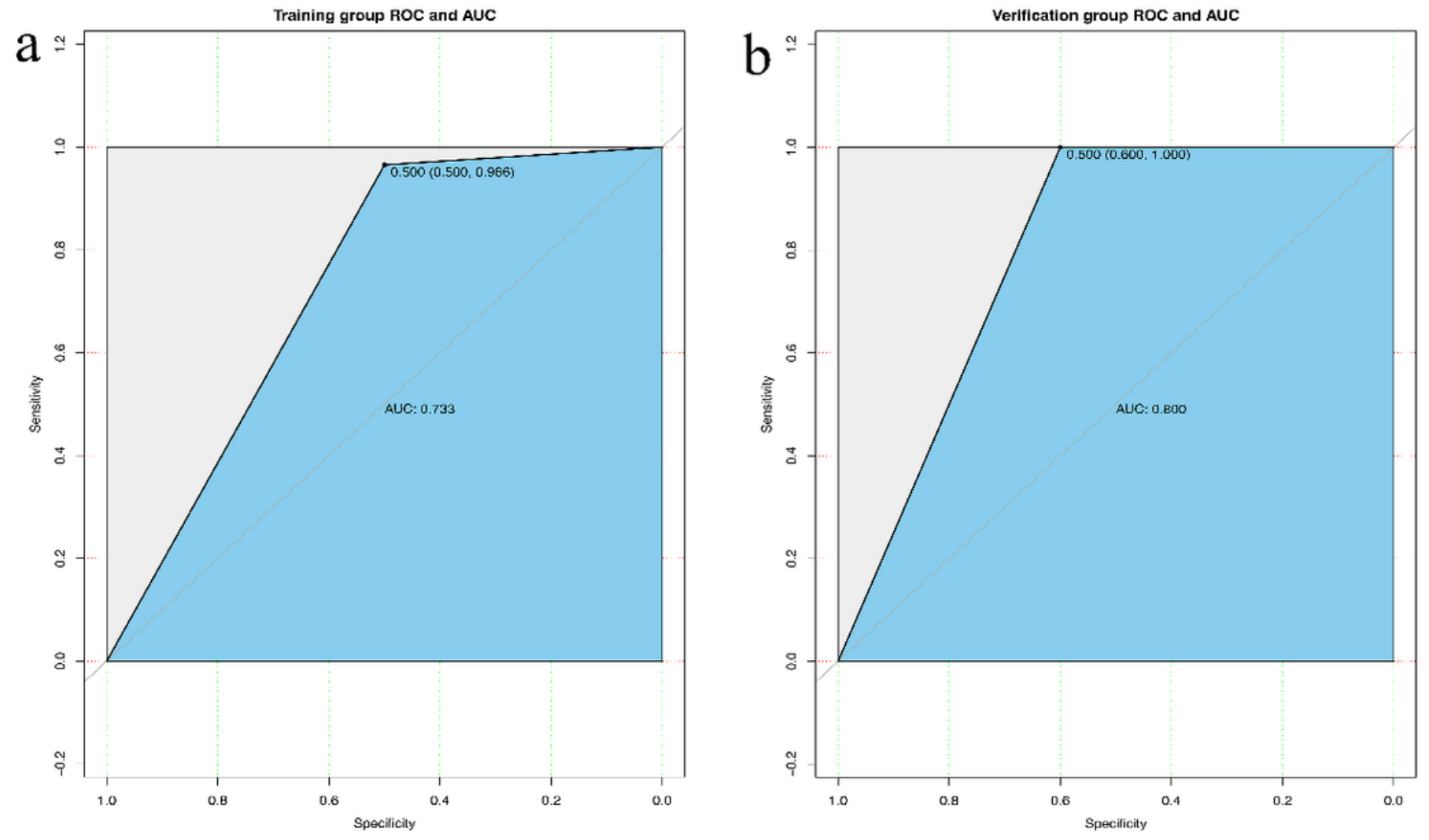
Figure 6

ROC curve analysis of training set (a) and verification set (b) in the AdaBoost ML model
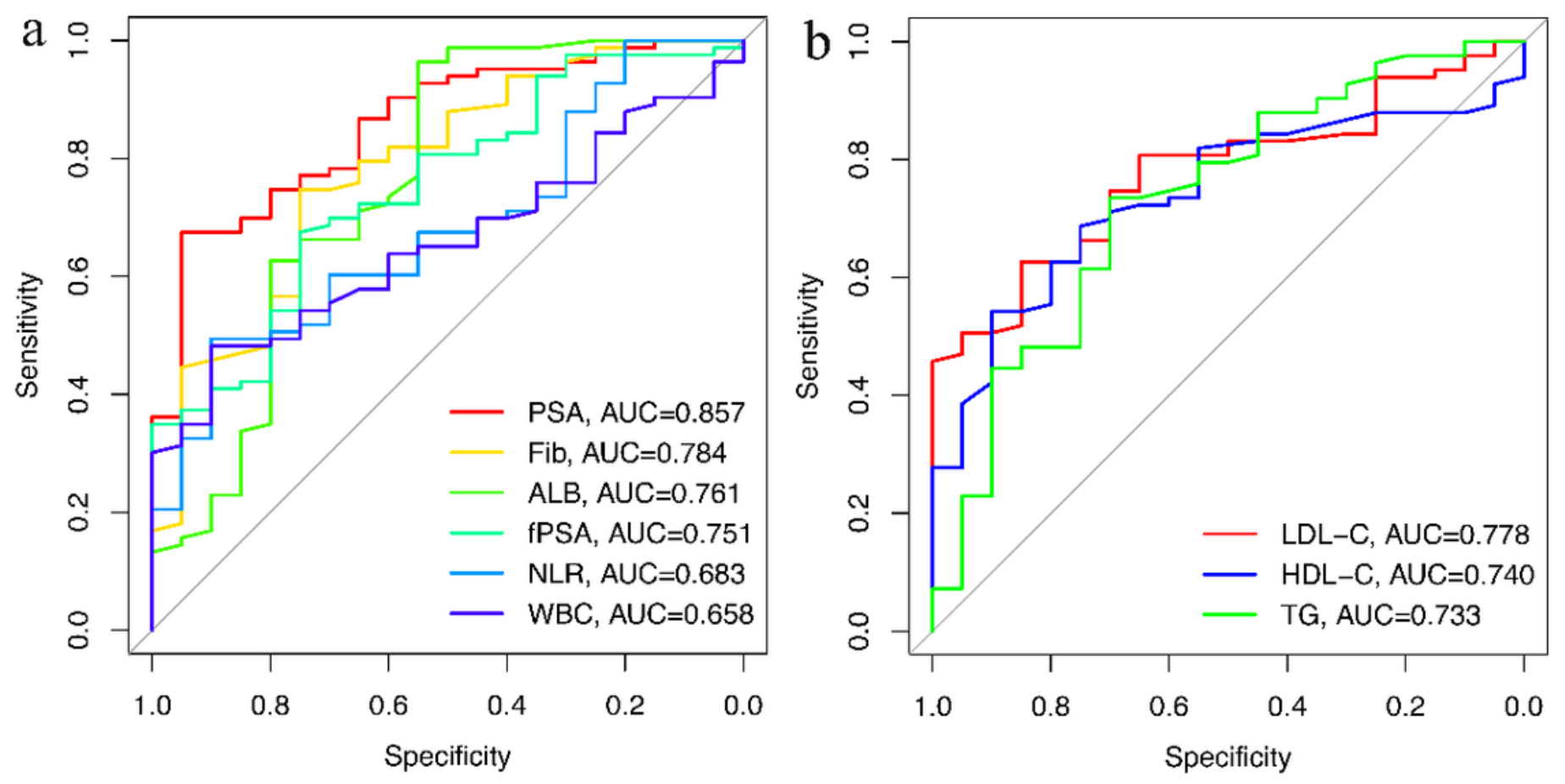

Figure 7

a ROC curves of PSA and systemic inflammation indicators; b ROC curve of metabolic indicators 

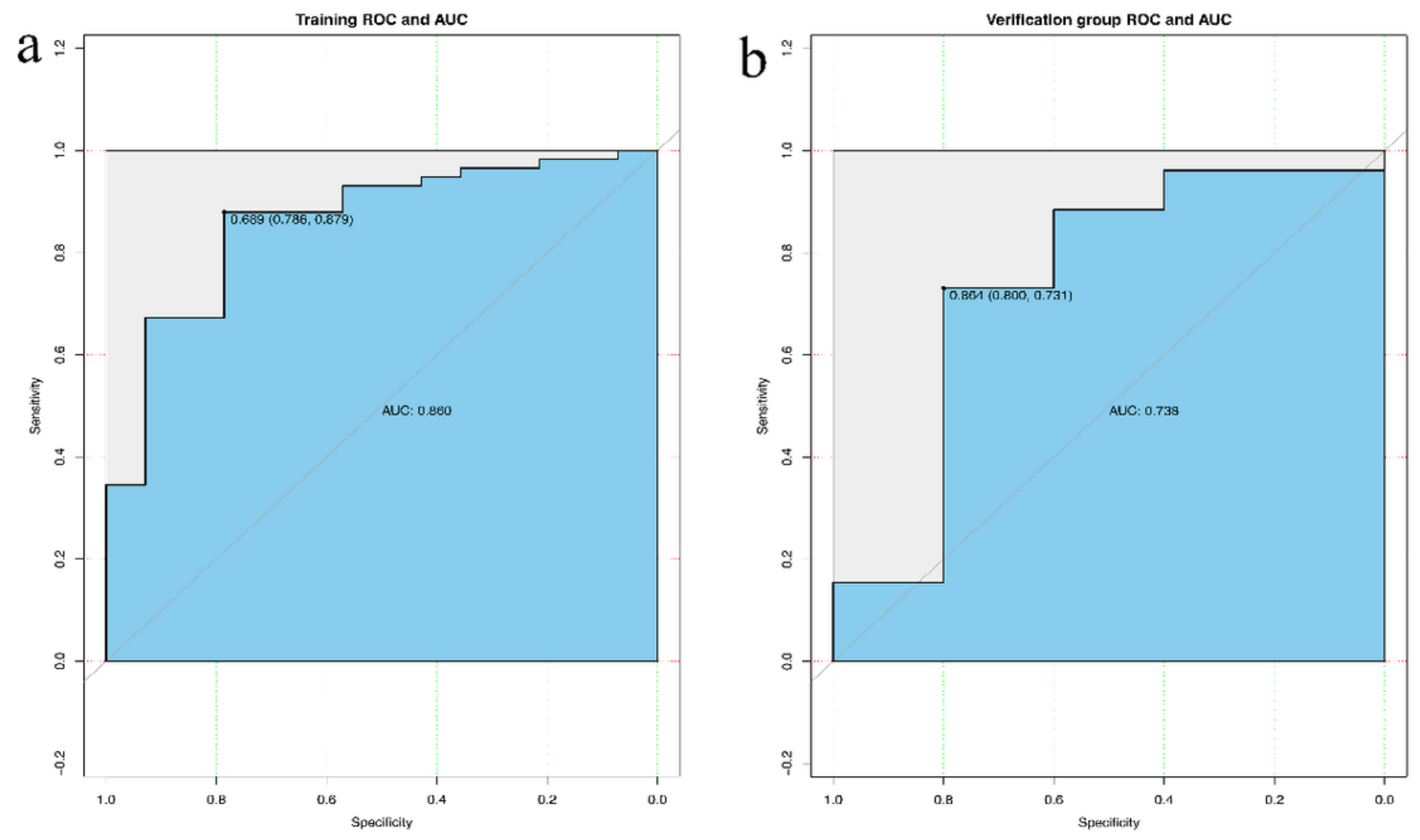

Figure 8

ROC curve analysis of training group (a) and verification group (b) in C-Logistic classification model 


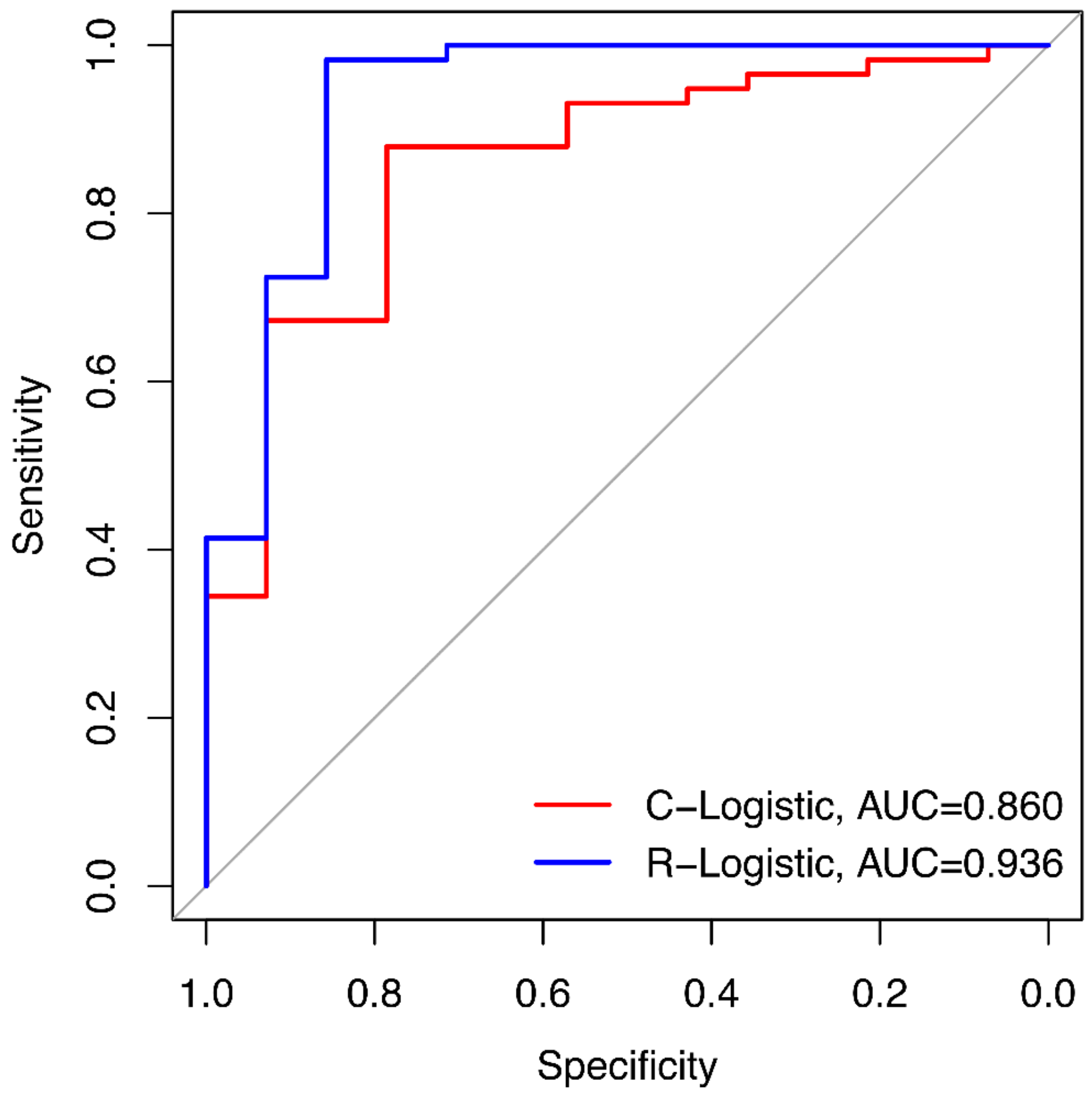

Figure 9

Comparison of ROC curves between R-logistic and C-logistic 
Points

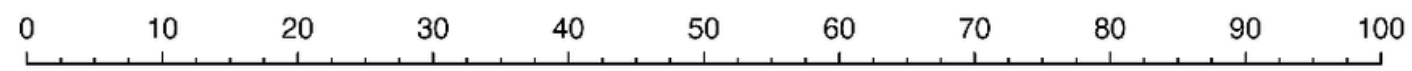

Y(radiomics)

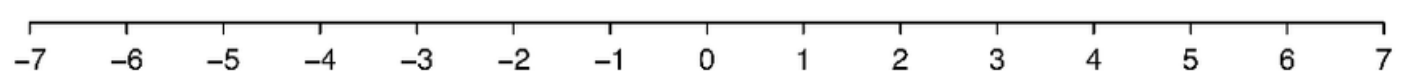

Total Points

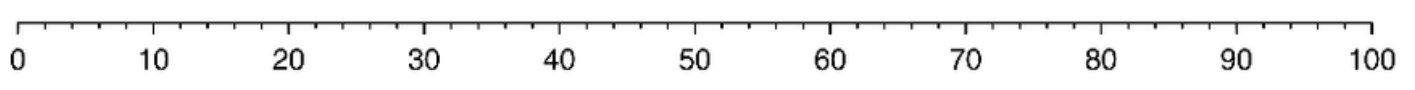

Risk of $\mathrm{PCa}$

$\begin{array}{llllll}0.1 & 0.2 & 0.4 & 0.6 & 0.8 & 0.9\end{array}$

\section{Figure 10}

Nomogram of imagomics 


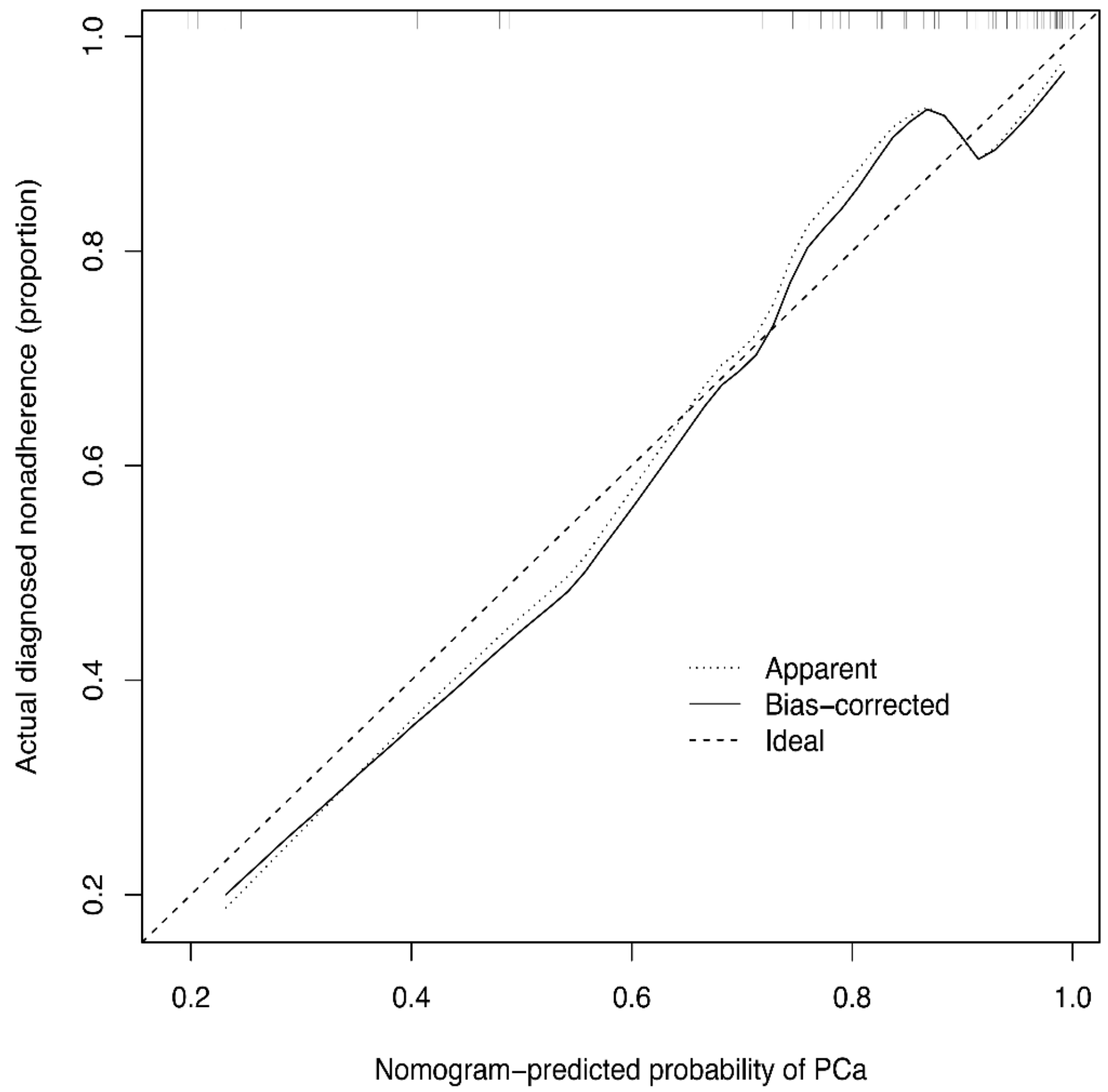

Figure 11

Nomogram calibration curve 